\title{
Clinical Characteristics and Prognostic Factors of Children with Guillain-Barré Syndrome
}

\author{
Sea Hyung Kim, MD', Furkat Samadov, MD ${ }^{1,2}$, Akmal Mukhamedov, MD ${ }^{1,2}$, Juhyun Kong, MD ${ }^{1,3}$, Ara Ko, MD ${ }^{1,3}$, \\ Young Mi Kim, MD ${ }^{4}$ Yun-Jin Lee, MD ${ }^{1,3}$, Sang Ook Nam, MD ${ }^{1,3}$ \\ 1 Division of Pediatric Neurology, Department of Pediatrics, Pusan National University Children's Hospital, Pusan National University School \\ of Medicine, Yangsan, Korea \\ ${ }^{2}$ Neuroscience Center, National Children's Medical Center, Tashkent, Uzbekistan \\ ${ }^{3}$ Research Institute for Convergence of Biomedical Science and Technology, Pusan National University Yangsan Hospital, Yangsan, Korea \\ ${ }^{4}$ Department of Pediatrics, Pusan National University Hospital, Pusan National University School of Medicine, Busan, Korea
}

Received: August 16, 2019

Revised: October 10, 2019

Accepted: October 10, 2019

Corresponding author:

Ara Ko

Department of Pediatrics, Pusan

National University Children's

Hospital, Pusan National University

School of Medicine, 20 Geumo-ro,

Mulgeum-eup, Yangsan 50612,

Korea

Tel: $+82-55-360-2180$

Fax: +82-55-360-2181

E-mail: chereara@gmail.com
Purpose: Guillain-Barré syndrome (GBS) is a group of clinical syndromes involving acute polyneuropathy secondary to an immune-mediated process. Although affected children generally tend to have a good prognosis, a small subset of children experience residual symptoms that require lengthy and costly rehabilitation. Therefore, we aimed to describe the clinical findings of children with GBS and to investigate factors associated with their outcomes.

Methods: A retrospective study was conducted of previously healthy children $\leq 18$ years of age diagnosed with GBS and admitted to Pusan National Children's Hospital between 2009 and 2018.

Results: Of the 38 patients included in the study (23 boys [60.5\%]; median age, 4.3 years [interquartile range, 2.8 to 9.7 years]), 28 (73.7\%) fully recovered without any residual symptoms, while $10(26.3 \%)$ had residual symptoms more than 6 months after the onset of GBS. The presence of autonomic dysfunction and a $\geq 50 \%$ decrease in compound muscle action potential (CMAP) amplitude from the lower limit of normal were associated with functional deficits at 6 months. Age, sex, type of antecedent illness, cranial nerve involvement, the need for mechanical ventilation, and the subtype of GBS were not significantly associated with functional outcomes. Post-gadolinium enhancement of nerve roots was seen in $71.1 \%$ of patients on initial spine magnetic resonance imaging (MRI).

Conclusion: Contrast enhancement of nerve roots on MRI was the most helpful initial finding for diagnosing GBS. The presence of autonomic dysfunction and $a \geq 50 \%$ decrease in CMAP from the lower limit of normal were poor prognostic factors for functional outcomes.

Keywords: Guillain-Barre syndrome; Child; Prognosis

\section{Introduction}

Guillain-Barré syndrome (GBS) refers to a group of clinical syndromes with acute polyneuropathy secondary to an immune-me- diated process, which usually presents with progressive weakness that can involve autonomic, bulbar, and respiratory systems, and reduced or diminished deep tendon reflexes [1]. Since the eradication of polio, GBS has become the most frequent cause of acute or

Copyright(C) 2019 Korean Child Neurology Society

This is an Open Access article distributed under the terms of the Creative Commons Attribution Non-Commercial License (http://creativecommons.org/licenses/by-nc/4.0/) which permits unrestricted non-commercial use, distribution, and reproduction in any medium, provided the original work is properly cited. 
subacute flaccid weakness, and reported annual incidence rates of GBS in children range from 0.34 to 1.34 per 100,000 [1-10].

GBS is a clinical diagnosis that can be made when progressive weaknesses in limbs and areflexia or hyporeflexia are present [11]. Other supporting features include progression of symptoms lasting up to 4 weeks, relative symmetry of weakness and sensory loss, less impressive sensory symptoms than weakness if present, pain in back and legs, autonomic dysfunction, absence of fever, albuminocytologic dissociation in cerebrospinal fluid (CSF) studies, and post-gadolinium enhancement of peripheral nerve roots and cauda equina in magnetic resonance imaging (MRI) [12]. Acute inflammatory demyelinating polyneuropathy (AIDP), which targets segments of myelin sheath, is the most common clinical subtype of GBS, along with less common subtypes such as acute motor axonal neuropathy (AMAN) and acute motor and sensory axonal neuropathy (AMSAN) which target neuronal axon, and Miller-Fischer syndrome that presents with ataxia, areflexia, and ophthalmoplegia $[11,13]$.

The differential diagnosis of the presenting symptoms of GBS flaccid paralysis, areflexia, or a combination-is extensive including pathology of cerebellum, spinal cord, peripheral nerves, and muscles, and often lead to extensive and costly medical investigations [11]. Children tend to have a better prognosis than adults, with about $80 \%$ of children showing full recovery without longterm recurrence [11]. However, a small subset of children experiences residual symptoms that require lengthy and costly rehabilitation. Therefore, in this study, we described clinical characteristics of children with GBS with the aim of searching for findings that can aid in making the diagnosis, and early predictive factors for achieving full recovery.

\section{Materials and Methods}

Retrograde chart review was done for previously healthy children of $\leq 18$ years of age diagnosed with GBS and admitted to Pusan National Children's Hospital between 2009 and 2018. Patients were included in the study when recorded outcome following 6 months after onset were available. Patients who showed disease progression beyond 4 weeks, who had the previous history of other neurologic or chronic systemic diseases, or who were transferred from other hospitals during the course of the disease were excluded.

Diagnosis of GBS was made clinically in patients showing progressive weakness of more than one limb and areflexia/hyporeflexia, according to criteria suggested by Asbury and Cornblath [14]. Detailed neurologic examination findings in a standardized manner, hematological findings, CSF findings, electrophysiological findings, and spine MRI findings were available in all patients. Antecedent febrile illnesses were all recorded. If the predominant associating symptoms were respiratory symptoms including cough, sputum, and rhinorrhea, it was classified as respiratory antecedent illness. If the predominant accompanying symptoms were gastrointestinal symptoms including vomiting or diarrhea, it was classified as gastrointestinal illness. Details on history of antecedent vaccinations were not complete and therefore were not included.

Hematologic and CSF investigations were all done on the 1st day of admission. Data of neutrophil, lymphocyte, and platelet counts, sodium, albumin, erythrocyte sedimentation rate, C-reactive protein (CRP), creatine kinase, free thyroxine, and thyroid stimulating hormone (TSH) on blood tests, and CSF protein were available in all patients. Neutrophil/lymphocyte ratio (NLR) and platelet/lymphocyte ratio (PLR) were calculated by dividing neutrophil or platelet counts by lymphocyte counts, respectively.

Electrophysiological and MRI investigations were all done within 48 hours since admission except for two patients whose spine MRIs were taken 10 and 13 days after admission respectively, due to unstable conditions requiring intensive care unit care. When the above investigations were repeated, initial findings were collected for analysis. However, for classification of subtypes, subsequent electrophysiological abnormal findings were used.

Sub-classifications of AIDP, AMAN, and AMSAN were made based on electrophysiological criteria: (1) AIDP when evidence of demyelination (prolongation of distal latency, slow nerve conduction velocity, f-wave prolongation, conduction block, and temporal dispersion) was observed; (2) AMAN when compound muscle action potential (CMAP) was reduced without evidence of demyelination; and (3) AMSAN when reduced CMAP and sensory nerve action potential amplitudes were observed [15]. Patients were classified as having Miller-Fisher syndrome when they showed low-amplitude sensory nerve action potentials together with the clinical triad of ataxia, areflexia, and ophthalmoplegia [15]. Patients whose electrophysiological results were repeatedly normal were classified as unclassifiable.

Patients were divided into two groups according to their outcome at 6 months - those who achieved full recovery and those with residual motor deficits. Other residual functional deficits present at 6 months were also collected, including bladder dysfunction. Subjective symptoms that are not retractable from records of physical examinations during follow-up visits were not included due to incompleteness of data.

Statistical analysis was performed using SPSS version Subscription 2019 (IBM Co., Armonk, NY, USA). Data from statistical analyses are expressed as medians and interquartile ranges (IQRs) for continuous and ordinal variables and as counts and percentages 
for categorical variables. Univariate analysis for factors affecting functional outcome was done using binary logistic regression. A $P$ value of $<0.05$ was considered significant.

This study was approved by the Institutional Review Board of Pusan National University Yangsan Hospital (IRB no. 05-2019176). Written informed consents from patients were waived due to a retrospective nature of the study.

\section{Results}

A total of 38 children who had been previously healthy and subsequently diagnosed with GBS were subject to this study. The clinical characteristics of the patients are summarized in Table 1 . The median age of onset was 4.3 years (IQR, 2.8 to 9.7), and more than half $(55.3 \%)$ of patients had onset before 5 years of age. Twenty-three $(60.5 \%)$ were boys, and seasonal distribution showed peak prevalence during winter $(42.1 \%)$, and least prevalence during autumn (7.9\%). Sensory change or pain was accompanied in $19(50.0 \%)$ of patients, whereas autonomic dysfunction was seen in six (15.8\%) patients (five with urinary dysfunction and one with both urinary and cardiovascular dysfunctions), and cranial nerve involvements were seen in four patients (10.5\%). Three patients $(7.9 \%)$ were admitted to the pediatric intensive care unit and were supported with mechanical ventilation. Albuminocytologic dissociations in CSF were present in 14 patients (36.8\%) on the first day of admission. Eleven of the 24 patients whose previous CSF findings were negative repeated the CSF exam after 7 to 18 days, which revealed albuminocytologic dissociations in eight patients.

Spine MRIs with T1-weighted, T2-weighted, and post-gadolinium images of the whole spine were done in all patients, and contrast enhancement of spinal nerve roots was present in 27 patients (71.1\%). Two patients were evaluated with spine MRI after 48 hours since admission due to their unstable conditions. Of the 36 patients who were evaluated with spine MRI within 48 hours since admission, 25 patients (69.4\%) showed contrast enhancement. Subgroup classification showed 23 patients (60.5\%) with ADIP, 10 (26.3\%) with AMAN, one (2.6\%) with AMSAN, and two (5.3\%) with Miller-Fisher syndrome. The rest two patients whose initial and follow-up electrophysiological studies were all normal remained as unclassifiable.

When outcomes of 6 months after the onset were assessed, 28 patients $(73.7 \%)$ were fully recovered without any motor residual symptoms, while 10 patients $(26.3 \%)$ still had motor residual symptoms. No patient died during 6 months after the onset. Among 10 patients with residual motor weakness, six patients had not achieved independent walking while four patients had weak-
Table 1. Clinical characteristics of 38 children with Guillain-Barré syndrome

\begin{tabular}{|c|c|}
\hline Clinical variable & No. of patients (\%) \\
\hline \multicolumn{2}{|l|}{ Age (yr) } \\
\hline$<1$ & 0 \\
\hline $1-4.9$ & $21(55.3)$ \\
\hline $5-9.9$ & $9(23.7)$ \\
\hline $10-18$ & $8(21.1)$ \\
\hline \multicolumn{2}{|l|}{ Sex } \\
\hline Boys & $23(60.5)$ \\
\hline Girls & $15(39.5)$ \\
\hline \multicolumn{2}{|l|}{ Season } \\
\hline Spring (March-May) & $10(26.3)$ \\
\hline Summer (June-August) & $9(23.7)$ \\
\hline Autumn (September-November) & $3(7.9)$ \\
\hline Winter (December-February) & $16(42.1)$ \\
\hline \multicolumn{2}{|l|}{ Antecedent illness } \\
\hline Respiratory & $21(55.3)$ \\
\hline Gastrointestinal & $4(10.5)$ \\
\hline Other febrile illnesses & $10(26.3)$ \\
\hline None & $3(7.9)$ \\
\hline Sensory change/Pain & $19(50.0)$ \\
\hline Autonomic dysfunction & $6(15.8)$ \\
\hline Cranial nerve involvement & $4(10.5)$ \\
\hline Mechanical ventilation & $3(7.9)$ \\
\hline Albuminocytologic dissociation in CSF & $14(36.8)$ \\
\hline Contrast enhancement in spine MRI & $27(71.1)$ \\
\hline \multicolumn{2}{|l|}{ Subtypes } \\
\hline AIDP & $23(60.5)$ \\
\hline AMAN & $10(26.3)$ \\
\hline AMSAN & $1(2.6)$ \\
\hline Miller-Fisher & $2(5.3)$ \\
\hline Unclassifiable & $2(5.3)$ \\
\hline \multicolumn{2}{|l|}{ Outcome after 6 months } \\
\hline Full recovery & $28(73.7)$ \\
\hline Residual symptoms & $10(26.3)$ \\
\hline Incomplete independent gait & $6(15.8)$ \\
\hline $\begin{array}{l}\text { Motor weakness with ability to walk inde- } \\
\text { pendently }\end{array}$ & $4(10.5)$ \\
\hline Death & 0 \\
\hline
\end{tabular}

CSF, cerebrospinal fluid; MRI, magnetic resonance imaging; AIDP, acute inflammatory demyelinating polyneuropathy; AMAN, acute motor axonal neuropathy; AMSAN, acute motor and sensory axonal neuropathy.

ness in limbs or truncal instability but could walk independently. Three of six patients (7.9\% of total) who did not achieve independent gait also showed bladder dysfunction necessitating clean intermittent catheterizations. One of four patients ( $2.6 \%$ of total) with milder weakness who achieved independent gait also showed concomitant facial palsy.

Intravenous immunoglobulin (IVIG) of $2 \mathrm{mg} / \mathrm{kg}$ over 3 to 5 
days was given to all patients during admission. In patients who did not show initial response to IVIG with progressing symptoms, plasma exchange was tried in two patients and intravenous methylprednisolone of $30 \mathrm{mg} / \mathrm{kg}$ for 3 days was tried in three patients. The two of the three patients who received intravenous methylprednisolone achieved full recovery later, while the rest one patient and two patients who underwent plasma exchange showed residu- al symptoms after 6 months.

After dividing patients into two groups - one group with 28 patients who achieved the full recovery and another group with 10 patients with residual motor deficits - clinical characteristics were compared between the two groups in search for clinical factors affecting the clinical outcome (Table 2). Among clinical factors listed in Table 2, presence of autonomic dysfunction (odds ratio [OR],

Table 2. Univariate analysis of clinical factors and functional outcome at 6 months

\begin{tabular}{|c|c|c|c|c|}
\hline Clinical variable & Full recovery $(n=28)$ & Functional deficit $(n=10)$ & OR $(95 \% \mathrm{Cl})$ & $P$ value \\
\hline Age (yr) & $3.5(2.6-8.0)$ & $8.1(3.0-13.4)$ & $1.125(0.969-1.306)$ & 0.122 \\
\hline Sex (boys) & $18(64.3)$ & $5(50.0)$ & $0.556(0.129-2.394)$ & 0.430 \\
\hline Seasons & & & & 0.518 \\
\hline Spring & $8(28.6)$ & $2(20.0)$ & Reference group & \\
\hline Summer & $7(25.0)$ & $2(20.0)$ & $1.143(0.126-10.386)$ & \\
\hline Autumn & $1(3.6)$ & $2(20.0)$ & $8.000(0.45-139.290)$ & \\
\hline Winter & $12(42.9)$ & $4(40.0)$ & $1.333(0.196-9.083)$ & \\
\hline Antecedent illness & & & & 0.457 \\
\hline Respiratory & $14(50.0)$ & $7(70.0)$ & Reference group & \\
\hline Others & $12(42.9)$ & $2(20.0)$ & $0.333(0.058-1.919)$ & \\
\hline None & $2(7.1)$ & $1(10.0)$ & $1.000(0.077-13.016)$ & \\
\hline Symptom onset to admission (day) & $3.0(1.0-6.0)$ & $3.5(2.0-14.0)$ & $1.064(0.946-1.196)$ & 0.301 \\
\hline Sensory change/Pain & $13(46.4)$ & $6(60.0)$ & $1.731(0.399-7.505)$ & 0.464 \\
\hline Autonomic dysfunction & $1(3.6)$ & $5(50.0)$ & 27.000 (2.576-282.979) & 0.006 \\
\hline Cranial nerve involvement & $3(10.7)$ & $1(10.0)$ & $0.926(0.085-10.085)$ & 0.950 \\
\hline Mechanical ventilation & $1(3.6)$ & $2(20.0)$ & $6.750(0.539-84.464)$ & 0.139 \\
\hline Contrast enhancement in spine MRI & $18(64.3)$ & $9(90.0)$ & $5.000(0.551-45.391)$ & 0.153 \\
\hline CSF protein (mg/dL) & $32.8(15.0-90.0)$ & $73.0(19.3-154.4)$ & $1.001(0.995-1.006)$ & 0.790 \\
\hline Neutrophil $(/ \mu \mathrm{L})$ & $5,503(2,539-8,240)$ & $6,323(3,097-9,072)$ & $1.000(1.000-1.000)$ & 0.640 \\
\hline Lymphocyte $(/ \mu \mathrm{L})$ & $3,547(2,165-4,383)$ & $2,787(1,294-4,637)$ & $1.000(0.999-1.000)$ & 0.260 \\
\hline Platelet (109/L) & $400(302-473)$ & $369(308-513)$ & $1.002(0.995-1.008)$ & 0.597 \\
\hline NLR & $1.38(0.47-3.03)$ & $2.43(0.64-5.27)$ & $1.072(0.850-1.351)$ & 0.558 \\
\hline PLR & $112.7(78.5-149.1)$ & $129(84-256)$ & $1.001(0.997-1.005)$ & 0.472 \\
\hline CK (U/L) & $74.0(58.0-121.0)$ & $87.0(46.5-126.5)$ & $1.004(0.996-1.013)$ & 0.304 \\
\hline Sodium (mmol/L) & $137.0(134.3-138.0)$ & $137.3(137.5-142.3)$ & $1.269(0.979-1.645)$ & 0.072 \\
\hline Albumin (g/dL) & $4.55(4.18-4.70)$ & $4.40(4.13-4.63)$ & $0.866(0.346-2.167)$ & 0.759 \\
\hline $\mathrm{ESR}(\mathrm{mm} / \mathrm{hr})$ & $9.0(4.0-14.0)$ & $11.5(4.5-46.8)$ & $1.055(0.948-1.174)$ & 0.324 \\
\hline $\mathrm{CRP}(\mathrm{mg} / \mathrm{dL})$ & $0.09(0.01-0.20)$ & $0.12(0.02-0.48)$ & $14.702(0.315-685.420)$ & 0.170 \\
\hline Free T4 (ng/dL) & $1.32(1.14-1.37)$ & $1.25(1.00-1.42)$ & $0.042(0.002-23.569)$ & 0.327 \\
\hline TSH (mIU/L) & $1.37(0.66-2.43)$ & $1.96(1.09-2.76)$ & $1.406(0.643-3.073)$ & 0.393 \\
\hline \multicolumn{5}{|l|}{ Subtypes } \\
\hline AIDP & $15(53.6)$ & 8 (80.0) & Reference group & \\
\hline Others & $13(46.4)$ & $2(20.0)$ & $0.288(0.052-1.608)$ & 0.156 \\
\hline CMAP amplitude & & & & 0.010 \\
\hline Normal or $\geq 50$ LLN & $22(78.6)$ & $3(30.0)$ & Reference group & \\
\hline$<50$ LLN or absent & $6(21.4)$ & $7(70.0)$ & 8.556 (1.683-43.495) & \\
\hline
\end{tabular}

Values are presented as median (interquartile range) or number (\%).

$\mathrm{OR}$, odds ratio; $\mathrm{Cl}$, confidence interval; $\mathrm{MRI}$, magnetic resonance imaging; CSF, cerebrospinal fluid; NLR, neutrophil/lymphocyte ratio; PLR, platelet/ lymphocyte ratio; CK, creatin kinase; ESR, erythrocyte sedimentation rate; CRP, C-reactive protein; T4, thyroxine; TSH, thyroid stimulating hormone; AIDP, acute inflammatory demyelinating polyneuropathy; CMAP, compound muscle action potential; LLN, lower limit of normal. 
27.000; 95\% confidence interval [CI], 2.576 to $282.979 ; P=0.006$ ) and absent or decrease in CMAP amplitude to $<50 \%$ of lower limit of normal (LLN) (OR, 8.556; 95\% CI, 1.683 to $43.495 ; P=0.010)$ were only factors that were significantly associated with increased risk of poor clinical outcome after 6 months.

Multivariate analysis was performed with the parameters that demonstrated $P$ values of $<0.020$ - age, presence of autonomic dysfunction, use of mechanical ventilation, presence of contrast enhancement on spine MRI, plasma sodium level, plasma CRP level, and CMAP amplitude. Absent or decreased CMAP amplitude was the only clinical factor significantly associated with poor outcome on multivariate analysis (OR, $273.879 ; 95 \% \mathrm{CI}, 0.417$ to $179,920.480 ; P=0.039)$.

\section{Discussion}

In this study, apart from findings obtained from detailed history taking and physical examinations, contrast enhancement of peripheral nerve roots and cauda equina on MRI was the most helpful initial finding for making the diagnosis of GBS. Contrast enhancements were seen in $71.1 \%(27 / 38)$ of total patients on their initial spine MRIs, and in $69.4 \%$ (25/36) of patients whose MRIs were taken within 48 hours of admission. After 6 months of admission, $26.3 \%$ of patients had residual symptoms. When looking at predictive factors of this functional outcome after 6 months, presence of autonomic dysfunction and $\geq 50 \%$ decrease in CMAP amplitude from LLN were related to the presence of functional deficit.

Although GBS is the cause for the majority of patients presenting with acute or subacute flaccid paralysis, the incidence is rare as stated before and even rarer in children. The age group known to be most commonly affected in children is 1 to 4 years of age [16]. Around $70 \%$ of patients have identified previous infections including cytomegalovirus, Mycoplasma pneumoniae, Epstein-Barr virus, influenza A, Haemophilus influenzae, enterovirus, and Campylobacter jejuni, and the most commonly associated organism identified is Campylobacter jejuni whose antibodies are found in about $40 \%$ of patients $[3,12,17,18]$. Previous studies have shown the high occurrence of GBS winter and summer regarding seasonal distribution which is possibly due to the higher occurrence of associated infections in these seasons, especially gastrointestinal infections during summer [19-22]. In this study, age group of 1 to 4.9 years was most commonly affected (55.3\%), as in previous studies. Winter season showed peak incidence (42.1\%) in accordance with existing studies, but summer did not show a distinct peak. Also, while higher proportions of patients had the history of antecedent illness (92.1\%) than in previous reports, a much smaller subset of patients had gastrointestinal infections (10.5\%). Retrospective na- ture of this study may be attributable to this discrepancy. As laboratory investigations for associated organisms were only done in a small subset of patients included in this study, history from parents or caregivers were analyzed and most parents reported their children to have had "common cold" for previous infections that were mostly of benign nature.

The prognosis of children is known to be better than adults possibly due to shorter nerve length or better nerve regeneration in children, but there is a great paucity of studies regarding the prognosis of GBS in the pediatric population [23]. In a prospective study of 95 children with GBS including two patients later diagnosed with chronic inflammatory demyelinating polyneuropathy, $75 \%$ of the children were symptom-free at last follow-up without any mortality (follow-up duration of 10 to 604 days, median 288 days) [24]. In another prospective study with 324 patients, the prognosis was better with $96 \%$ of patients achieving independent gait, but also showed $1.5 \%$ of mortality [25]. The proportion of patients who achieved independent gait but showed minor functional disability was not mentioned [25]. In this study, $73.7 \%$ of patients showed full recovery after 6 months, $15.8 \%$ did not achieve independent walking, and $10.5 \%$ achieved independent gait but showed minor functional disabilities after 6 months. After 1 year, one patient who could not walk independently achieved independent gait, and two patients with minor motor weakness became symptom-free, resulting in $78.9 \%$ of patients with full recovery, and $13.2 \%$ of patients without the ability to walk independently after 1 year.

Numerous factors have been suggested as prognostic factors of GBS in studies mostly subjecting adults, and severe deficits at onset, cranial nerve involvement, autonomic nerve involvement, requiring mechanical ventilation, preceding diarrhea, short interval between symptom onset and admission, absent/low amplitude CMAP or axonal lesion patterns in electrophysiological studies are examples of suggested poor prognostic factors [26-28]. Higher age was also a poor prognostic factor in adults [26,27]. However, in children, younger age was associated with poor outcome regarding the achievement of independent gait [25]. In a study which compared the outcome of demyelinating and axonal forms of GBS in children, there was no difference among different subtypes [29]. In an effort for earlier prediction of outcome, biomarkers that associate with the outcome have been searched, and several acute phase markers such as low albumin, low sodium, high NLR, high PLR, and high CRP levels have been shown to be related to poor prognosis of GBS in adults [30-33]. Also, patients with GBS showed lower levels of TSH correlating with disease severity in adults [34]. In this study, autonomic dysfunction and absent or low CMAP of $\geq 50 \%$ of LLN were factors associated with poor functional out- 
come. None of the acute phase markers such as high NLR, high PLR, high CRP, hyponatremia, low albumin, or low TSH were associated with clinical outcome in this study, suggesting that these markers are not readily applicable prognostic factors in children.

This study has significance as it contributes to the scarce volume of literature describing possible prognostic factors of GBS in children, and also investigated implications for acute phase biomarkers in children. However, it has several limitations including the retrospective nature of this study and the small number of included patients.

\section{Conflicts of interest}

No potential conflict of interest relevant to this article was reported.

\section{ORCID}

Sea Hyung Kim, https://orcid.org/0000-0002-1162-7314

Ara Ko, https://orcid.org/0000-0002-3008-8432

\section{Author contributions}

Conceptualization: AK. Data curation: SHK, FS, AM, and JK. Formal analysis: AK. Funding acquisition: AK. Methodology: AK. Project administration: AK. Visualization: SHK. Writing-original draft: SHK and AK. Writing-review \& editing: AK, YMK, YJL, and SON.

\section{Acknowledgements}

This study was supported by a 2018 research grant from Pusan National University Yangsan Hospital.

\section{References}

1. McGrogan A, Madle GC, Seaman HE, de Vries CS. The epidemiology of Guillain-Barre syndrome worldwide: a systematic literature review. Neuroepidemiology 2009;32:150-63.

2. Sipila JOT, Soilu-Hanninen M, Ruuskanen JO, Rautava P, Kyto V. Epidemiology of Guillain-Barre syndrome in Finland 20042014. J Peripher Nerv Syst 2017;22:440-5.

3. Willison HJ, Jacobs BC, van Doorn PA. Guillain-Barre syndrome. Lancet 2016;388:717-27.

4. Rantala H, Uhari M, Niemela M. Occurrence, clinical manifestations, and prognosis of Guillain-Barre syndrome. Arch Dis Child 1991;66:706-9.
5. Barzegar M, Dastgiri S, Karegarmaher MH, Varshochiani A. Epidemiology of childhood Guillan-Barre syndrome in the north west of Iran. BMC Neurol 2007;7:22.

6. Dias-Tosta E, Kuckelhaus CS. Guillain Barre syndrome in a population less than 15 years old in Brazil. Arq Neuropsiquiatr 2002;60(2-B):367-73.

7. Hart DE, Rojas LA, Rosario JA, Recalde H, Roman GC. Childhood Guillain-Barre syndrome in Paraguay, 1990 to 1991. Ann Neurol 1994;36:859-63.

8. Hung KL, Wang HS, Liou WY, Mak SC, Chi CS, Shen EY, et al. Guillain-Barre syndrome in children: a cooperative study in Taiwan. Brain Dev 1994;16:204-8.

9. Rantala H, Cherry JD, Shields WD, Uhari M. Epidemiology of Guillain-Barre syndrome in children: relationship of oral polio vaccine administration to occurrence. J Pediatr 1994;124:2203.

10. Ismail EA, Shabani IS, Badawi M, Sanaa H, Madi S, Al-Tawari A, et al. An epidemiologic, clinical, and therapeutic study of childhood Guillain-Barre syndrome in Kuwait: is it related to the oral polio vaccine? J Child Neurol 1998;13:488-92.

11. Chung A, Deimling M. Guillain-Barre syndrome. Pediatr Rev 2018;39:53-54.

12. Donofrio PD. Guillain-Barre syndrome. Continuum (Minneap Minn) 2017;23(5, Peripheral Nerve and Motor Neuron Disorders):1295-309.

13. Hughes RA, Rees JH. Clinical and epidemiologic features of Guillain-Barre syndrome. J Infect Dis 1997;176 Suppl 2:S92-8.

14. Asbury AK, Cornblath DR. Assessment of current diagnostic criteria for Guillain-Barre syndrome. Ann Neurol 1990;27 Suppl:S21-4.

15. Cornblath DR. Electrophysiology in Guillain-Barre syndrome. Ann Neurol 1990;27 Suppl:S17-20.

16. Landaverde JM, Danovaro-Holliday MC, Trumbo SP, Pacis-Tirso CL, Ruiz-Matus C. Guillain-Barre syndrome in children aged $<15$ years in Latin America and the Caribbean: baseline rates in the context of the influenza A (H1N1) pandemic. J Infect Dis 2010;201:746-50.

17. Lehmann HC, Hartung HP, Kieseier BC, Hughes RA. Guillain-Barre syndrome after exposure to influenza virus. Lancet Infect Dis 2010;10:643-51.

18. Van den Berg B, Walgaard C, Drenthen J, Fokke C, Jacobs BC, van Doorn PA. Guillain-Barre syndrome: pathogenesis, diagnosis, treatment and prognosis. Nat Rev Neurol 2014;10:469-82.

19. Sankhyan N, Sharma S, Konanki R, Gulati S. Childhood Guillain-Barre syndrome subtypes in northern India. J Clin Neurosci 2014;21:427-30.

20. Lin JJ, Hsia SH, Wang HS, Lyu RK, Chou ML, Hung PC, et al. 
Clinical variants of Guillain-Barre syndrome in children. Pediatr Neurol 2012;47:91-6.

21. Benamer HT, Bredan A. Guillain-Barre syndrome in Arab countries: a systematic review. J Neurol Sci 2014;343:221-3.

22. Van Doorn PA, Kuitwaard K, Walgaard C, van Koningsveld R, Ruts L, Jacobs BC. IVIG treatment and prognosis in Guillain-Barre syndrome. J Clin Immunol 2010;30 Suppl 1:S74-8.

23. Delanoe C, Sebire G, Landrieu P, Huault G, Metral S. Acute inflammatory demyelinating polyradiculopathy in children: clinical and electrodiagnostic studies. Ann Neurol 1998;44:350-6.

24. Korinthenberg R, Schessl J, Kirschner J. Clinical presentation and course of childhood Guillain-Barre syndrome: a prospective multicentre study. Neuropediatrics 2007;38:10-7.

25. Barzegar M, Toopchizadeh V, Maher MHK, Sadeghi P, Jahanjoo F, Pishgahi A. Predictive factors for achieving independent walking in children with Guillain-Barre syndrome. Pediatr Res 2017;82:333-9.

26. Gonzalez-Suarez I, Sanz-Gallego I, Rodriguez de Rivera FJ, Arpa J. Guillain-Barre syndrome: natural history and prognostic factors: a retrospective review of 106 cases. BMC Neurol 2013; 13:95.

27. Rajabally YA, Uncini A. Outcome and its predictors in Guillain-Barre syndrome. J Neurol Neurosurg Psychiatry 2012;83:
711-8.

28. Walgaard C, Lingsma HF, Ruts L, van Doorn PA, Steyerberg EW, Jacobs BC. Early recognition of poor prognosis in Guillain-Barre syndrome. Neurology 2011;76:968-75.

29. Karalok ZS, Taskin BD, Yanginlar ZB, Gurkas E, Guven A, Degerliyurt A, et al. Guillain-Barre syndrome in children: subtypes and outcome. Childs Nerv Syst 2018;34:2291-7.

30. Ozdemir HH. Analysis of the albumin level, neutrophil-lymphocyte ratio, and platelet-lymphocyte ratio in Guillain-Barre syndrome. Arq Neuropsiquiatr 2016;74:718-22.

31. Sahin S, Cinar N, Karsidag S. Are cerebrospinal fluid protein levels and plasma neutrophil/lymphocyte ratio associated with prognosis of Guillain Barre syndrome? Neurol Int 2017;9:7032.

32. Vaishnavi C, Kapoor P, Behura C, Singh SK, Prabhakar S. C-reactive protein in patients with Guillain Barre syndrome. Indian J Pathol Microbiol 2014;57:51-4.

33. Tunc A. Early predictors of functional disability in Guillain-Barre syndrome. Acta Neurol Belg 2019 Apr 8 [Epub]. https://doi.org/10.1007/s13760-019-01133-3.

34. Huang Y, Ying Z, Chen Z, Xiang W, Su Z, Quan W, et al. Thyroid hormone level is associated with the frequency and severity of Guillain-Barre syndrome. Int J Neurosci 2017;127:893-9. 